\title{
Observer-based Economic Model Predictive Control for Direct Contact Membrane Distillation
}

\author{
Xingang Guo, Fahad Albalawi, and Taous-Meriem Laleg-Kirati
}

\begin{abstract}
In this work, we present an Observer-based Economic Model Predictive Control (OEMPC) scheme for Direct Contact Membrane Distillation (DCMD). To compute control actions via MPC strategy, full knowledge of the system states is necessary to predict the future evolution of the system. However, the full state measurement for DMCD is either difficult or costly. In this work, an integrated framework of an observer design and EMPC paradigm is proposed for the DMCD process. Numerical simulations are presented to illustrate the performance of the proposed observer design. Closed-loop simulations show that the proposed OEMPC scheme was able to operate the process economically while satisfying process and input constraints.
\end{abstract}

Index Terms-Direct Contact Membrane Distillation (DCMD), Observer-based Economic Model Predictive Control (OEMPC), Observer design, Differential Algebraic Equations (DAEs).

\section{INTRODUCTION}

$\mathbf{T}$ HE lack of clean fresh water is one of the most pervasive problems affecting human life around the world. There are several natural sources such as rivers, groundwater and water conversion, that can provide fresh water less costly. However, these natural water sources are not always available, and the amount of water contained in them constitute only small percentage of the global water distribution [9]. Desalination plants as an alternative way to produce freshwater have recently attracted water researcher's attention since the begin of $21^{\text {th }}$ century. To achieve sustainable desalination process, renewable energy sources such as solar and wind energy can be used to transform seawater into distilled water. The main two methods of desalination techniques seawater using solar energy are thermal-based desalination techniques and the mechanical-based desalination. Membrane Distillation (MD) is a thermally driven desalination process that has been developed in 1960 [7, 30]. However, successful industrial implementation of the MD for large scale plants has not emerged yet due to the operational challenges associated with the MD technology. For example, the low distilled water production and the presence of membrane fouling are the primary obstacles that prevent this technology from becoming

X.G. Guo and T.M. Laleg-Kirati are with Computer, Electrical and Mathematical Sciences and Engineering Division, King Abdullah University of Science and Technology, Thuwal 23955-6900, Saudi Arabia, e-mail: ( \{xingang.guo, taousmeriem.laleg\}@kaust.edu.sa).

F. Albalawi is with Electrical Engineering Department, Taif University, Taif 26571, Saudi Arabia e-mail: (f.alboloi@tu.edu.sa). F. Albalawi is also with Computer, Electrical and Mathematical Sciences and Engineering Division, King Abdullah University of Science and Technology, Thuwal 23955-6900, Saudi Arabia, e-mail: (fahad.albalawi@kaust.edu.sa). F. Albalawi is with Department of Electrical and Electronic Engineering, Imperial College London, SW7, 2AZ, UK, e-mail: (F.albalawi@imperial.ac.uk). commercially available. There are several types of MD technologies such as Air Gap Membrane Distillation (AGMD), Vacuum Membrane Distillation (VMD), Permeate Gap Membrane Distillation (PGMD), and Direct Contact Membrane Distillation (DCMD). In this work, we consider the DCMD process depicted in Fig. 1 in which both the feed and permeate solutions are kept in direct contact with the membrane [28].

Model Predictive Control (MPC) is an advanced control paradigm that utilizes the dynamic model of the process to compute the optimal control actions while satisfying several operational constraints. MPC has been widely used in water desalination plants to both maximize the water production and meet input and states constraints. A two-layer modelbased control architecture was developed in [17] to optimally operate a Solar Membrane Distillation (SMD) system. In the upper layer, the MPC calculates offline temperature and flow rate set-points, and then PI and feed-forward controllers are implemented in the lower-layer to track these set-points. However, the proposed MPC scheme optimally drive the closed-loop system to the desired set-points without taking the inputs and process operational constraints into account. To overcome this, another MPC scheme was developed in [2] for a distributed solar-powered desalination plant, in which the proposed MPC strategy manipulated the flow rate to keep the temperature gradient value in the solar collectors as constant. Another form of MPC termed Economic Model Predictive Control (EMPC) was recently developed in [18] for the DCMD process to explicitly maximize the distilled water flux while meeting the input and several operational restrictions. We refer the readers to reference [13] for more details on the EMPC design.

All aforementioned MPC paradigms incorporate an explicit mathematical model of the controlled process. The modeling for DCMD process is not new, researchers have developed several mathematical models for this process. For instance, Partial Differential Equation (PDE)-based models was derived in $[6,10]$. Nevertheless, developing MPC paradigms for nonlinear system modeled by PDEs is is computationally heavy and is not satiable for real implementation. Alternatively, [23] derived a nonlinear Differential Algebraic Equation (DAE)based model for the DCMD system. As a reduced-order of PDE model, this DAE model is more computation efficient and has been validated experimentally [22]. In this work, we will utilize this DAE-based model to develop the MPC algorithm for DCMD process.

For any MPC design, full knowledge of the process states is required to initiate the process model used in the MPC formulation. State-estimated-based EMPC was studied in [14, 20] 
for a class of nonlinear systems, where the high gain observer is used to estimate the system state using output measurements. In addition, a Robust Moving Horizon Estimation (RMHE) scheme and EMPC are combined in [24] to achieve the optimal process economic performance with closed-loop stability. In this work, full state measurement for DCMD is either difficult to obtain or costly. Therefore, designing an observer for DCMD processes is required so that optimal controllers such as MPC can be implemented. A first attempt has been proposed in $[11,12,16]$ where a nonlinear observer was developed for the DCMD process modeled by PDEs. However, the PDE model is not computation efficient as mentioned above. Therefore, in this paper, we consider the DCMD process modeled by DAEs proposed in [23], and propose an observer design based on this DAE model. In the past two decades, observer design was proposed for various classes of DAE systems. For instance, an observer design was proposed for linear descriptor systems in [8, 21] where sufficient conditions for detectability and observability were given. In addition, nonlinear observer design was proposed in $[1,3,5]$ for nonlinear DAE systems. In [26], full order and reduced-order observer designs were developed for continuous-time descriptor systems with Lipschitz constraint. Inspired by the observer design in [26], we propose a full order nonlinear observer for DCMD based on Linear Matrix Inequality (LMI). Unlike most of the observers developed for DAE systems, the proposed observer design guarantees exponential convergence of the estimation error to zero by satisfying sufficient conditions that are independent of any coordinate transformation.

Motivated by the above considerations, we propose an Observer-based Economic Model Predictive (OEMPC) scheme for the DCMD process. The OEMPC combines both EMPC and a nonlinear observer. The nonlinear observer estimates the system states based on the measurements, and the EMPC obtains the observer estimates to predict the future evolution of the process so that optimal control actions can be computed. The proposed OEMPC aims to maximize the distilled water flux while reducing energy consumption. This paper is organized as follows. In section II, the DAE model of DCMD system will be introduced briefly and followed by the EMPC formulation for DCMD system in section III. Section IV formulates the states estimation problem and develops the nonlinear observer for DCMD process. Numerical simulations also show the performance of the proposed observer in this section. The OEMPC formulation and implementation methods will be discussed in section V. Section VI shows the closed-loop simulation results and followed by the conclusion VII.

\section{Differential Algebraic Equations for Direct CONTACT MEMBRANE Distillation}

In this work, we consider the nonlinear DAE-based model derived in [23] for the DCMD process of Fig. 1. In this model, the DCMD module composed of series of control-volume cells to consider spatial variations on the temperature along the feed and permeate flow directions. To do so, an analogous electrical

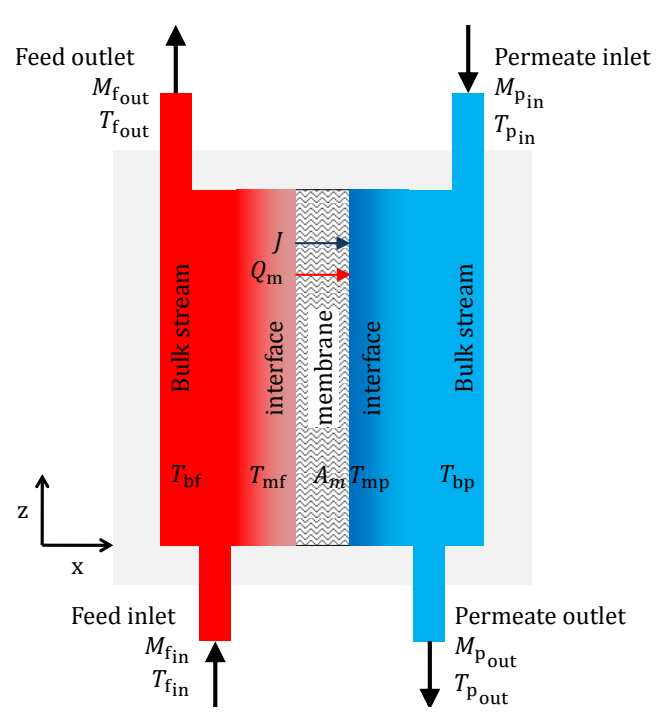

Fig. 1: Diagrammatic sketch of DCMD module [23].

thermal network for each cell is designed and its elements are parameterized. Using the lumped capacitance method, a dynamical DAE-based model for the DCMD is constructed as follows [23]:

$$
\begin{aligned}
E \dot{x} & =F(x, u) x+B(u) \\
y & =C x,
\end{aligned}
$$

where $x \in \Omega \subset R^{6 \mathrm{~N}_{c}+4}$ denotes both differential states and algebraic states $\left(4 \mathrm{~N}_{c}+2\right.$ differential states and $2 \mathrm{~N}_{c}+2$ algebraic states) and $\mathrm{N}_{c}$ is the number of control-volume cells chosen to model the system, $\Omega$ is the domain of the process states, which is not the whole space due to the physical constraints. System states are defined in the following order:

$$
\begin{gathered}
x=\left[Q_{\mathrm{f}_{1}}, T_{\mathrm{bf}_{1}}, \cdots, Q_{\mathrm{f}_{\mathrm{N}_{\mathrm{c}}}}, T_{\mathrm{bf}_{\mathrm{N}_{c}}}, Q_{\mathrm{f}_{\mathrm{N}_{c+1}}}, Q_{\mathrm{p}_{1}}, T_{\mathrm{bp}_{1}}\right. \\
, \cdots, Q_{\mathrm{p}_{\mathrm{N}_{c}}}, T_{\mathrm{bp}_{\mathrm{N}_{c}}}, Q_{\mathrm{p}_{\mathrm{N}_{c+1}}}, T_{\mathrm{f}_{\text {out }}}, T_{\mathrm{p}_{\text {out }}}, \\
\left.T_{\mathrm{mf}_{1}}, \cdots, T_{\mathrm{mf}_{\mathrm{N}_{c}}}, T_{\mathrm{mp}_{1}}, \cdots, T_{\mathrm{mp}_{\mathrm{N}_{c}}}\right] .
\end{gathered}
$$

The heat transfer rate into and out of the $n_{\text {th }}$ feed cell are represented by $Q_{\mathrm{f}_{\mathrm{n}}}$ and $Q_{\mathrm{f}_{\mathrm{n}+1}}$, respectively. Similarly, the heat transfer rate into and out of the $n_{\text {th }}$ permeate cell are denoted by $Q_{\mathrm{p}_{\mathrm{n}}}$ and $Q_{\mathrm{p}_{\mathrm{n}+1}}$, respectively. $T_{\mathrm{bf}_{\mathrm{n}}}$ and $T_{\mathrm{bp}_{\mathrm{n}}}$ are the bulk temperatures which are assumed to be uniform through the cell except at the membrane interface due to the temperature polarization effect. $T_{\mathrm{mf}_{\mathrm{n}}}$ and $T_{\mathrm{mp}_{\mathrm{n}}}$ represent the feed side and permeate side interface temperature of the $n_{\text {th }}$ cell, respectively. The outlet temperature of the feed side and permeate side are denoted by $T_{\mathrm{f}_{\mathrm{out}}}$ and $T_{\mathrm{p}_{\mathrm{out}}}$, respectively.

The nonlinear dynamics of Eq. 1 is depicted in the matrix $F(x, u) \in R^{\left(6 \mathrm{~N}_{c}+4\right) \times\left(6 \mathrm{~N}_{c}+4\right)}$ where it is composed of several block matrices as follows:

$$
F(x, u)=\left[\begin{array}{ccccc}
A_{f}(x, u) & \mathbf{0} & Z_{f_{1}}(x, u) & Z_{f_{2}} & \mathbf{0} \\
\mathbf{0} & A_{p}(x, u) & Z_{p_{1}}(x, u) & \mathbf{0} & Z_{p_{2}}(x) \\
T_{f_{o}}(x) & T_{p_{o}}(x) & I & \mathbf{0} & \mathbf{0} \\
Z_{1}(x) & Z_{2} & \mathbf{0} & Z_{3} & Z_{4}(x) \\
\mathbf{0} & Z_{5} & \mathbf{0} & Z_{6}(x) & Z_{7}(x)
\end{array}\right],
$$

The definition and dimensions of each the above block matrix can be found in [23]. The singular matrix of the nonlinear 
DAE model of Eq. 1 is denoted by $E$ and it is structured as follows:

$$
E=\left[\begin{array}{cc}
I_{\left(4 \mathrm{~N}_{c}+2\right) \times\left(4 \mathrm{~N}_{c}+2\right)} & \mathbf{0} \\
\mathbf{0} & \mathbf{0}_{\left(2 \mathrm{~N}_{c}+2\right) \times\left(2 \mathrm{~N}_{c}+2\right)}
\end{array}\right],
$$

where $I$ is the identity matrix and $\mathbf{0}$ is the zero matrix of the appropriate size.

The matrix $B(u) \in R^{\left(6 \mathrm{~N}_{c}+4\right) \times 2}$ represents the input channel to the system, which is a nonlinear function of which is given as follows:

$$
B(u)=\left[\begin{array}{cc}
8 \alpha_{1} M_{\mathrm{f}_{\mathrm{in}}}^{4} & 0 \\
0 & 0 \\
\vdots & \vdots \\
0 & 0 \\
0 & -8 \alpha_{2} M_{\mathrm{p}_{\text {in }}}^{4} \\
0 & 0 \\
\vdots & \vdots \\
0 & 0
\end{array}\right] \begin{aligned}
& 1 \\
& 4 \mathrm{~N}_{c}+1 \\
& 4 \mathrm{~N}_{c}+3 \\
& \vdots \\
& 6 \mathrm{~N}_{c}+4
\end{aligned}
$$

where $\alpha_{1}$ and $\alpha_{2}$ are constants. $M_{\mathrm{f}_{\text {in }}}$ and $M_{\mathrm{p}_{\mathrm{in}}}$ (see Figure 1) refer to the mass flow rate coming into the first cell in the feed side and permeate side, respectively. The feed inlet mass flow rate $M_{\mathrm{f}_{\mathrm{in}}}$ is the only manipulated input considered for this work. Hence the input $u(t)$ is defined as follows:

$$
u(t)=M_{\mathrm{f}_{\text {in }}} .
$$

The permeate inlet mass flow rate $M_{\mathrm{p}_{\text {in }}}$ and the permeate inlet temperature $T_{\mathrm{p}_{\text {in }}}$ are set as constants. As mentioned above, the DMCD system can be powered by solar energy as shown in Fig. 2. The seawater is heated by a solar collector and then stored in a feed tank. The hot seawater within the feed tank is pumped to the feed side of the DCMD, the outlet water of the feed side is recycled to the feed tank. The DCMD produce the distilled water which is stored in a freshwater tank. The cold water within the freshwater tank is also pumped as the inlet water of the permeate side of the DCMD module. To mitigate the realistic behavior of the variation of the solar radiation along the day, we vary the feed inlet temperature value $T_{\mathrm{f}_{\mathrm{in}}}$ during the simulation time. One possible realization of that can be seen in Fig. 3.

The output of the system is the feed outlet temperature and permeate outlet temperature as follows:

$$
y(t)=\left[\begin{array}{l}
T_{\mathrm{f}_{\text {out }}} \\
T_{\mathrm{p}_{\text {out }}}
\end{array}\right] .
$$

The corresponding $C \in R^{2 \times\left(6 \mathrm{~N}_{c}+4\right)}$ matrix is given as follows:

$$
C=\left[\begin{array}{lll}
\mathbf{0}_{2 \times 4 \mathrm{~N}_{c}+2} & I_{2 \times 2} & \mathbf{0}_{2 \times 2 \mathrm{~N}_{c}}
\end{array}\right]
$$

This model captures the spatial and temporal responses of the temperature distribution along the flow direction. Also, the model is able to accurately predict the distilled water flux output based on the experimental validation in [22, 23]. (more details about the DAE model of Eq. 1 can be found in $[22,23]$ ).

\section{ECONOMIC MOdEL PREDICTIVE CONTROL FOR DCMD PROCESS}

In this section, we derive EMPC paradigm for the DCMD process of Eq. 1. In the first subsection, we introduce the objective function for DCMD process to formulate the EMPC, which aims to maximize the distilled water flux and reduce energy consumption. The second subsection proposes the EMPC design for DCMD process.

\section{A. Objective Function}

For DCMD process of Eq. 1, the feed inlet temperature $\left(T_{\mathrm{f}_{\text {in }}}\right)$ varies according to the solar radiation. This variation will affect the distilled water flux of the DCMD process. Figure 4 illustrates this relationship where the distilled water flux is measured under various feed inlet temperature values and different feed inlet mass flow rates. In this simulation, the permeate inlet mass flow rate was kept constant at 0.1111 $\mathrm{m} / \mathrm{s}$, while the feed inlet mass flow rate was increased from $0.1 \mathrm{~m} / \mathrm{s}$ to $0.73 \mathrm{~m} / \mathrm{s}$. This was repeated for 4 different feed inlet temperatures $\left(50^{\circ} \mathrm{C}, 60^{\circ} \mathrm{C}, 70^{\circ} \mathrm{C}\right.$, and $\left.80^{\circ} \mathrm{C}\right)$.

From Fig. 4, a proportional relationship between the distilled water flux and the feed inlet mass flow rates is observed where an increase in $T_{\mathrm{f}_{\text {in }}}$ induces an increase in the distilled water flux. Nevertheless, a saturation of the distilled water flux is observed at high feed inlet mass flow rate values as demonstrated in Fig. 4. Inspired by the above observations, the objective function $Y$ for the EMPC is chosen to maximize the distilled water flux $(J)$ while penalizes the feed inlet mass flow rate $u(t)$ as follows:

$$
\max Y=\max \beta_{1} J(x(t))-\beta_{2} u(t)
$$

where $\beta_{1}$ and $\beta_{2}$ are used to scale the terms of the objective function to be in the same order of magnitude. $J$ refers to the distilled water flux which can be expressed as follows:

$$
J=\zeta\left[\lambda \exp \left(23.2-\frac{227.02}{3816.4 T_{\mathrm{mf}}}\right)-\exp \left(23.2-\frac{227.02}{3816.4 T_{\mathrm{mp}}}\right)\right]
$$

where $\zeta$ and $\lambda$ refer to the membrane mass transfer coefficient and the mole fraction of $\mathrm{NaCl}$ in the feed stream, respectively. $T_{\mathrm{mf}}$ and $T_{\mathrm{mp}}$ are the temperatures at the membrane-feed interface and the membrane-permeate interface, respectively. After computing the values of Eq. 2 under different values feed inlet temperature, we observe that a maximum value of the objective function $(Y)$ exist for every choice of $T_{\mathrm{f}_{\mathrm{in}}}$ as shown in Fig. 5.Therefore, by choosing the objective function of the EMPC as Eq. 2, the optimization-based controller will try to track these optimal values while satisfying several operational constraints which will be given in the following subsection.

\section{B. EMPC Algorithm}

EMPC is an optimization-based control technique that maximizes an economical stage cost while meeting process and 


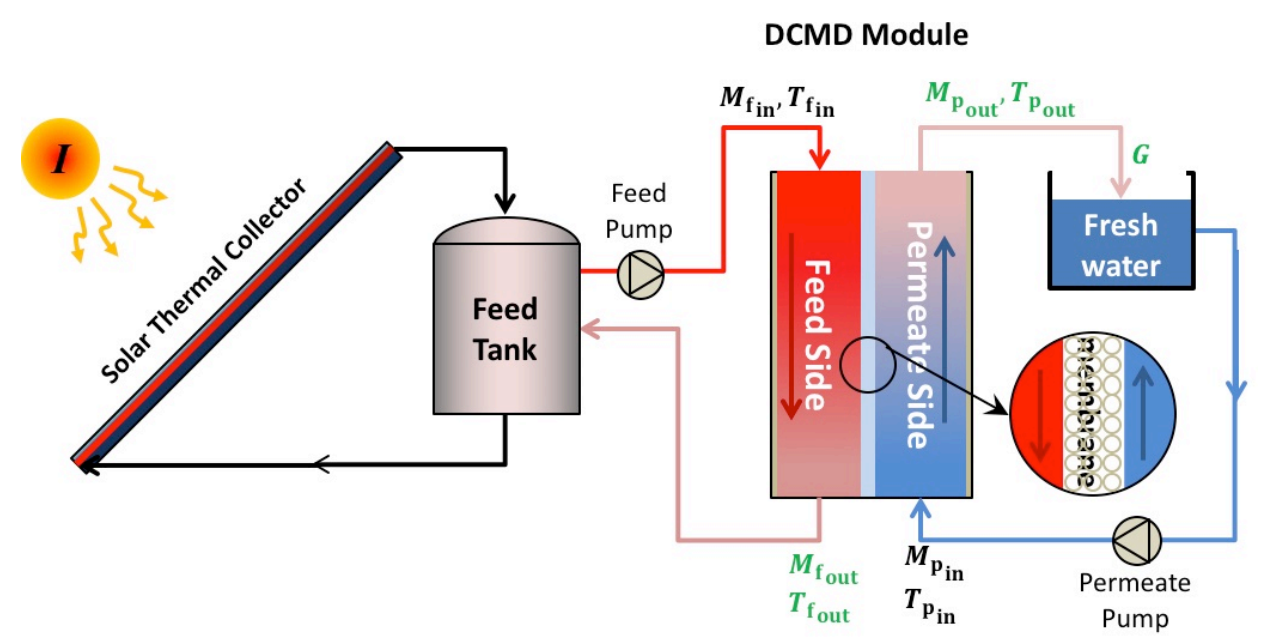

Fig. 2: Schematic diagram of Solar-powered DCMD module [4].

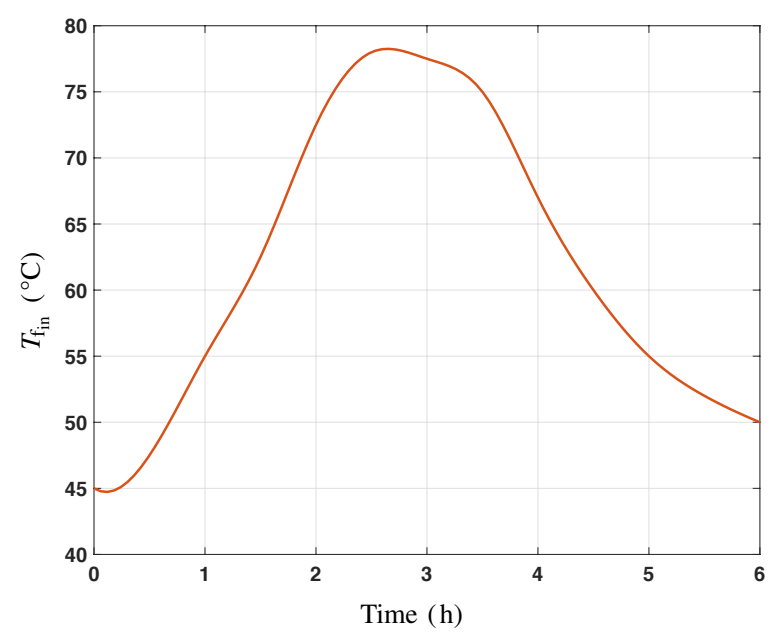

Fig. 3: The feed inlet temperature at feed side of the DCMD.

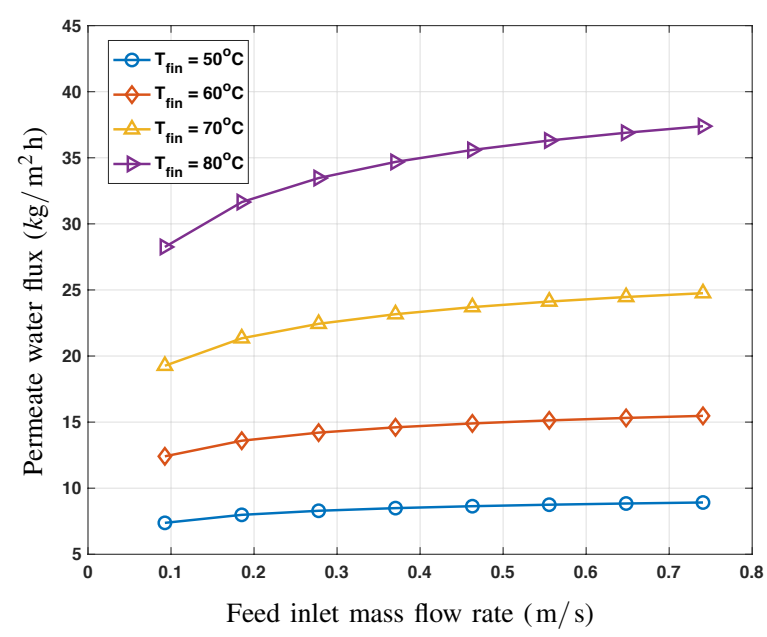

Fig. 4: The permeate flux respect to the feed inlet mass flow rate for various feed inlet temperatures.
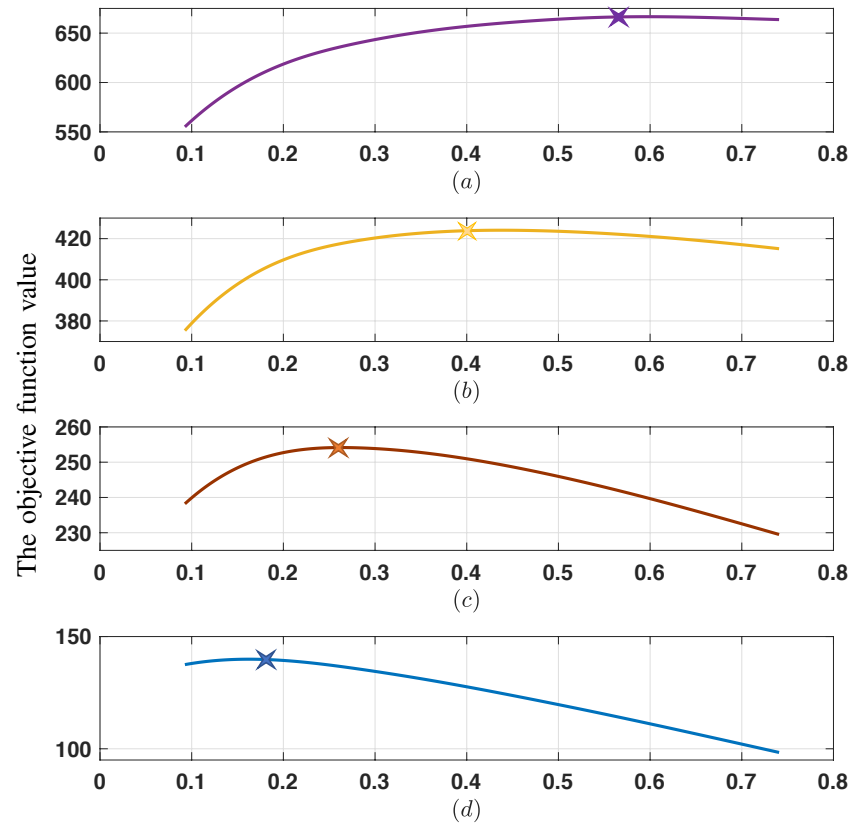

Feed inlet mass flow rate $(\mathrm{m} / \mathrm{s})$

Fig. 5: The objective function $Y$ for various feed inlet temperatures (a): the objective function value with feed inlet temperature $T_{\mathrm{f}_{\mathrm{in}}}=80^{\circ} \mathrm{C}$, (b): the objective function value with feed inlet temperature $T_{\mathrm{f}_{\mathrm{in}}}=70^{\circ} \mathrm{C}$, (c): the objective function value with feed inlet temperature $T_{\mathrm{f}_{\mathrm{in}}}=60^{\circ} \mathrm{C}$, (a): the objective function value with feed inlet temperature $T_{\mathrm{f}_{\mathrm{in}}}=50^{\circ} \mathrm{C}$.

input constraints. In order to achieve this, at each sampling 
time $t_{k}$, the following optimization problem is solved:

$$
\begin{aligned}
\max _{u(t) \in S(\Delta)} & \int_{t_{k}}^{t_{k+N}}\left[\beta_{1} J(\tilde{x}(\tau))-\beta_{2} u(t)\right] d \tau \\
\text { s.t. } & E \dot{\tilde{x}}=F(\tilde{x}, u) \tilde{x}+B(u) \\
& u(t) \in U, \forall t \in\left[t_{k}, t_{k+N}\right) \\
& \tilde{x}\left(t_{k}\right)=x\left(t_{k}\right) \\
& G(\tilde{x}) \leq \rho_{e}, \forall t \in\left[t_{k}, t_{k+N}\right) \\
& \left|u\left(t_{k}\right)-u\left(t_{k-1}\right)\right| \leq \varepsilon
\end{aligned}
$$

where the decision variable $u(t)$ denotes the manipulated input trajectory over the prediction horizon $N$. The family of piece-wise constant functions is signified by $S(\Delta)$. The manipulated input is restricted to be in a convex set over the prediction horizon $N \Delta(4 \mathrm{c})$ due to the physical constraints on the actuators. $U=\{u(t) \subset S(\Delta) \mid 0 \mathrm{~m} / \mathrm{s} \leq u \leq 2 \mathrm{~m} / \mathrm{s}\}$ is the convex set where the input values required to be within. The sampling period of the EMPC is denoted by $\Delta$, and the current sampling time is denoted by $t_{k}$. The control actions are implemented in a receding horizon fashion (i.e. only the first control action $u\left(t_{k}\right)$ is implemented, and then the EMPC horizon is rolled again over the next time step). Eq. 4a represents the objective function of the EMPC where the weighting parameter $\beta_{1}$ and $\beta_{2}$ are positive constants and chosen to scale the terms of the objective function to be in the same order of magnitude as discussed above. At the beginning of each sampling time $t_{k}$, the DAEs (Eq. $4 \mathrm{~b}$ ) is solved to predict the DCMD process state over the entire prediction horizon $N \Delta$ based on the state feedback at $t_{k}(\mathrm{Eq}$. $4 d)$, where the predicted states are denoted as $\tilde{x}(t)$. In this formulation for DCMD system, we enforce a boundedness constraint to keep the closed-loop state within a predefined stability region (Eq. 4e). The boundedness function is defined as $G(\tilde{x})=\left(\tilde{x}-x_{s s}\right)^{T} E^{T} P\left(\tilde{x}-x_{s s}\right)$ where $P$ is a positive definite matrix such that $E^{T} P \geq 0$. In our case, the $P$ is a diagonal positive definite matrix and is chosen to account for the different order of magnitude of the process states. Specifically, we set the matrix value that corresponds to heat transfer rate states to be equal to 0.01 , and 1 for the temperature states. $E$ is the singular matrix from DCMD system of Eq. 1. Finally, a smoothness constraint of Eq. 4f gives an upper bound of the difference between two consecutive control actions. Here we choose $\varepsilon$ to be equal to $0.5 \mathrm{~m} / \mathrm{s}$.

\section{State Estimation For DCMD System}

In this section, the full-order observer design of the DCMD system of Eq. 1 is first developed. In the next subsection, the influence of the estimation gain matrix $L$ on the differentiation index of the DAE-based observer is investigated. Finally, the simulation results are presented to illustrate the performance of the proposed observer for the DCMD system.

\section{A. Full-order Observer Design for DCMD}

To design the nonlinear observer for DCMD system of Eq. 1 , we first divide the nonlinear term $F(x, u)$ into a linear term and a nonlinear term. The DCMD model of Eq. 1 can be written as follows:

$$
\begin{aligned}
E \dot{x} & =A x+B(u)+\Phi(x, u), \\
y & =C x
\end{aligned}
$$

where $x \in \Omega$ is the system states. $A \in R^{\left(6 \mathrm{~N}_{c}+4\right) \times\left(6 \mathrm{~N}_{c}+4\right)}$ and $C \in$ $R^{2 \times\left(6 \mathrm{~N}_{c}+4\right)}$ are constant matrices. The nonlinear composition of the DCMD model of Eq. 1 is represented by $\Phi(x, u) \in$ $R^{6 \mathrm{~N}_{c}+4}$, which is continuously differentiable.

It is not difficult to show that $\Phi(x, u)$ is Lipschitz continuous since all the process states are physically bounded which ensure the boundness of Jacobain matrix of $\Phi(x, u)$. Hence $\Phi(x, u)$ is a Lipschitz function and satisfies the following Lipschitz condition for all $x$ and $\tilde{x} \in \Omega$ :

$$
\|\Phi(x, u)-\Phi(\tilde{x}, u)\| \leq \gamma\|x-\tilde{x}\|,
$$

where $\gamma$ is the Lipschitz constant.

Consider the following Luenberger-like full-order observer for Eq. 5 in a DAE form :

$$
\begin{aligned}
E \dot{\hat{x}} & =A \hat{x}+B(u)+\Phi(\hat{x})-L(y-C \hat{x}), \\
\hat{y} & =C \hat{x},
\end{aligned}
$$

where $\hat{x}$ is the estimated state vector and $L \in R^{\left(6 \mathrm{~N}_{c}+4\right) \times 2}$ is the observer gain matrix to be determined. Define the state estimation error as $e=x-\hat{x}$. By subtracting Eq. 5 from Eq. 7 , the following estimation error dynamic model is derived:

$$
E \dot{e}=(A+L C) e+\Phi(x, u)-\Phi(\hat{x}, u) .
$$

To guarantee exponential convergence of the estimation error to zero, we restate the following theorem from [26].

Theorem 1. There exists a full-order observer Eq. 7 for DCMD system Eq. 5 if there exist two matrices $P \in$ $R^{\left(6 N_{c}+4\right) \times\left(6 N_{c}+4\right)}$ and $Q \in R^{2 \times\left(6 N_{c}+4\right)}$ such that the following matrix inequalities are solvable:

$$
E^{T} P=P^{T} E \geq 0, \quad \Omega:=\left[\begin{array}{cc}
\Omega_{11} & P^{T} \\
P & -I
\end{array}\right]<0
$$

where $\Omega_{11}=A^{T} P+P^{T} A+C^{T} Q+Q^{T} C+\gamma^{2} I$.

If $Q$ and nonsingular matrix $P$ are solutions of (9), then the Eq. 7 with the following gain matrix:

$$
L=P^{-T} Q^{T}
$$

is a full-order observer for DCMD system 5. The proof of Theorem 1 can be found in [26].

Since Eq. 9 is not a strict LMI, in order to use LMI toolbox YALMIP [25], Eq. 9 needs to be transformed into an equivalent LMI. We use the following lemma which gives the strict LMI formulation [29]:

Lemma 1. The conditions in Theorem 1 are equivalent to the conditions that there exist a positive-definite matrix $X \in R^{6 N_{c}+4 \times 6 N_{c}+4}$, two matrices $Y \in R^{2 N_{c}+2 \times 6 N_{c}+4}$ and $Q \in$ $R^{2 \times 6 N_{c}+4}$ such that the following LMI is solvable:

$$
\left[\begin{array}{cc}
\Gamma & \left(X E+E_{\perp}^{T} Y\right)^{T} \\
\left(X E+E_{\perp}^{T} Y\right) & -I
\end{array}\right]<0,
$$


where $\Gamma=A^{T}\left(X E+E_{l}^{T} Y\right)+\left(X E+E_{\perp}^{T} Y\right)^{T} A+C^{T} Q+$ $Q^{T} C+\gamma^{2} I, \quad E_{\perp} \in R^{\left(2 N_{c}+2\right) \times\left(6 N_{c}+4\right)}$ satisfying $E_{\perp} E=0$ and $\operatorname{rank}\left(E_{\perp}\right)=2 N_{c}+2$.

For DCMD system, we can choose the following $E_{\perp}$ :

$$
E_{\perp}=\left[\begin{array}{ll}
\mathbf{0} & I
\end{array}\right],
$$

where $\mathbf{0} \in R^{\left(2 \mathrm{~N}_{c}+2\right) \times\left(4 \mathrm{~N}_{c}+2\right)}$ and $I \in R^{\left(2 \mathrm{~N}_{c}+2\right) \times\left(2 \mathrm{~N}_{c}+2\right)}$.

By means of Lemma 1, we have the following theorem which is a direct result from Theorem 1.

Theorem 2. There exists a full-order observer of Eq. 7 for $D C M D$ system of Eq. 5 if there exist a positive-definite matrix $X \in R^{\left(6 N_{c}+4\right) \times\left(6 N_{c}+4\right)}$, two matrices $Y \in R^{\left(2 N_{c}+2\right) \times\left(6 N_{c}+4\right)}$ and $Q \in R^{2 \times\left(6 N_{c}+4\right)}$ such that the LMI of Eq. 11 solvable.

We can compute the estimation gain matrix $L$ as follows:

$$
L=\left(X E+E_{\perp}^{T} Y\right)^{-T} Q^{T} .
$$

\section{B. Differentiation Index of the Observer System}

The differentiation index of a DAE system can be defined as the minimum number of times one has to differentiate the algebraic equations to get one differential equation for every algebraic state $[15,19]$. The index is an important indicator that qualitatively measures the complexity of solving DAE systems. DAEs with index one are numerically more stable than DAE systems with higher index. In [18], the authors proved that the DCMD system of Eq. 1 has an index one. However, since the correction term $-L(y(t)-C \hat{x}(t))$ appears in the observer system, the index of the DAE-based observer of Eq. 7 varies with respect to the estimation gain matrix. In this subsection, we evaluate the relationship between the index of the observer design of Eq. 7 and the estimation gain matrix $L$. To do so, we rewrite the observer system of Eq. 7 as follows:

$$
\left[\begin{array}{c}
\tilde{E} \\
\mathbf{0}
\end{array}\right] \dot{\hat{x}}(t)=\left[\begin{array}{l}
F_{d}(\hat{x}, u)-L_{d} C \\
F_{a}(\hat{x}, u)-L_{a} C
\end{array}\right] \hat{x}(t)+\left[\begin{array}{c}
B_{d}(u) \\
B_{a}
\end{array}\right]-\left[\begin{array}{l}
L_{d} \\
L_{a}
\end{array}\right] y(t) .
$$

The observer system is divided into two parts based on the differential states and algebraic states. $\tilde{E}$ is a full row rank matrix. $F_{d}(\hat{x}, u)$ and $F_{a}(\hat{x}, u)$ are the first $4 \mathrm{~N}_{c}+2$ rows and last $2 \mathrm{~N}_{c}+2$ rows of $F(\hat{x}(t), u)$, respectively. $L_{d}$ and $L_{a}$ are the first $4 \mathrm{~N}_{c}+2$ rows and last $2 \mathrm{~N}_{c}+2$ rows of the observer gain matrix $L . B_{d}(u)$ is a vector with the first $4 \mathrm{~N}_{c}+2$ elements of $B(u)$ and $B_{a}$ contains the remaining part, which is a constant matrix. After taking the time derivative of the algebraic part of Eq. 13 with respect to time $t$ (i.e., $\mathbf{0}=\left(F_{a}(\hat{x})-L_{a} C\right) \hat{x}(t)+$ $\left.B_{a} u(t)-L_{a} y(t)\right)$, we obtain the following system:

$$
\begin{gathered}
{\left[\begin{array}{c}
\tilde{E} \\
-F_{a}(\hat{x})+L_{a} C
\end{array}\right] \dot{\hat{x}}(t)=\left[\begin{array}{c}
F_{d}(\hat{x})-L_{d} C \\
F_{a}(\hat{x})
\end{array}\right] \hat{x}(t)+\left[\begin{array}{c}
B_{d}(u) \\
\mathbf{0}
\end{array}\right]} \\
-\left[\begin{array}{cc}
L_{d} & \mathbf{0} \\
\mathbf{0} & L_{a}
\end{array}\right]\left[\begin{array}{c}
y(t) \\
\dot{y}(t)
\end{array}\right] .
\end{gathered}
$$

The matrix $\left[\tilde{E}-F_{a}(\hat{x})+L_{a} C\right]^{T}$ may or may not be nonsingular due to the correction term $L_{a} C$. If this matrix is nonsingular, then the DAEs system of Eq. 1 is of index one. However, if $\left[\tilde{E}-F_{a}(\hat{x})+L_{a} C\right]^{T}$ is singular, then the DAEs of Eq. 1 is with higher index. The same procedure can be repeated until the non-singularity achieves. The number of times the algebraic equations need to be differentiated so that the matrix becomes nonsingular will be the index of the observer design of Eq. 7.

For the DCMD system, the $\left[\tilde{E}-F_{a}(\hat{x})+L_{a} C\right]^{T}$ matrix can be written as:

$$
\begin{gathered}
\text { written as: }\left[\begin{array}{c}
\tilde{E} \\
-F_{a}(\hat{x})+L_{a} C
\end{array}\right]= \\
\left.\begin{array}{ccccc}
I & \mathbf{0} & \mathbf{0} & \mathbf{0} & \mathbf{0} \\
\mathbf{0} & I & \mathbf{0} & \mathbf{0} & \mathbf{0} \\
-T_{f_{o}}(x) & -T_{p_{o}}(x) & -I+L_{a 1} & \mathbf{0} & \mathbf{0} \\
-Z_{1}(x) & -Z_{2} & L_{a 2} & -Z_{3} & -Z_{4}(x) \\
\mathbf{0} & -Z_{5} & L_{a 3} & -Z_{6}(x) & -Z_{7}(x)
\end{array}\right],
\end{gathered}
$$

where $L_{a 1} \in R^{2 \times 2}, L_{a 2} \in R^{\mathrm{N}_{c} \times 2}, L_{a 3} \in R^{\mathrm{N}_{c} \times 2}$ and

$$
\left[\begin{array}{l}
L_{a 1} \\
L_{a 2} \\
L_{a 3}
\end{array}\right]=L_{a} .
$$

It is trivial to show that the first $4 \mathrm{~N}_{c}+4$ rows of $\left[\tilde{E}-F_{a}(\hat{x})+\right.$ $\left.L_{a} C\right]^{T}$ are full row rank if and only if $-I+L_{a 1}$ is full rank.

We assume that

$$
L_{a 1}=\left[\begin{array}{ll}
p & q \\
m & n
\end{array}\right]
$$

then we have

$$
-I+L_{a 1}=\left[\begin{array}{cc}
p-1 & q \\
m & n-1
\end{array}\right] .
$$

The condition for $-I+L_{a 1}$ to be full rank is:

$$
(p-1)(n-1) \neq q m \text {. }
$$

For the last $2 \mathrm{~N}_{c}$ rows, the submatrix $S$ consisting of $-Z_{3}$, $-Z_{4}(x),-Z_{6}(x)$ and $-Z_{7}(x)$ in Eq. 15 is full row rank by tracing back to $[18,23]$, then the whole rectangular matrix is full row rank.

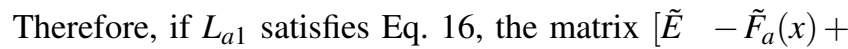
$\left.L_{a} C\right]^{T}$ is a nonsingular matrix. This indicates that the DAE system of Eq. 7 is of index one.

\section{Simulation results of the estimator for DCMD}

To demonstrate the performance of the proposed observer, numerical simulations of the actual system and the observer system are performed for a given input $u(t)$, as shown in Fig. 6. Square functions with different amplitudes are chosen as input $u(t)$. Even if this choice is not realistic, it allows to test the observer in a worse case scenario. In this simulations, the number of volume cells for both the system and the observer $\mathrm{N}_{c}=1$. This number of cells generates 10 differential and algebraic process states (6 differential states and 4 algebraic states). The permeate inlet temperature and the permeate inlet flow rate are fixed as $20^{\circ} \mathrm{C}$ and $0.1111 \mathrm{~m} / \mathrm{s}$, respectively. We use the designed feed inlet temperature as shown in Fig. 3.

As discussed earlier, it is important to select a gain matrix that satisfy the condition of Eq. 16 to ensure an index of one for our observer. To tests for several scenarios, we have changed the value of the Lipschitz constant $\gamma$ and computed the corresponding gain matrix by solving the LMI in Theorem 2. Table I shows the computation of gain matrix $L$ for different setting of $\mathrm{N}_{c}$ (number of cells) and $\gamma$. One can observe that as 


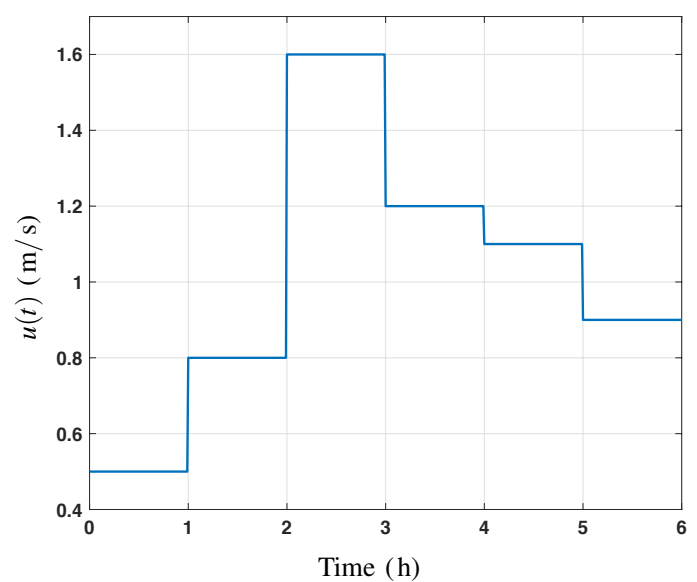

Fig. 6: The feed inlet mass flow rate of the DCMD.

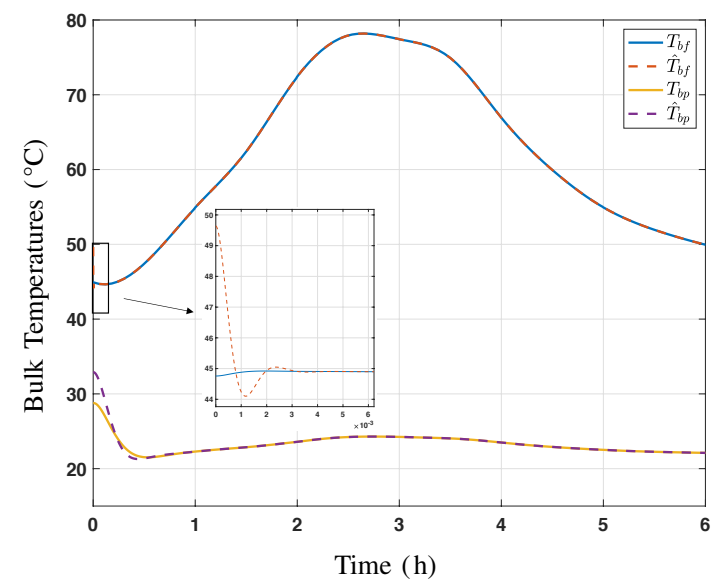

Fig. 7: The estimated and actual profile of bulk temperatures of the DCMD in feed side and permeate side.

$\mathrm{N}_{c}$ increases, the computational time to determine the gain matrix $L$ increases as well and inversely, the value of the Lipschitz constant decreases. This means that the LMI is less feasible when $\mathrm{N}_{c}$ increases, since the dimension of the matrices to be determined increases proportionally with the increase of $\mathrm{N}_{c}$.

TABLE I: Computation of observer gain matrix $L$

\begin{tabular}{clll}
$\mathrm{N}_{c}$ & \multicolumn{1}{c}{$\gamma$} & Computation Time (sec) & Condition of Eq. 16 \\
\hline \multirow{2}{*}{1} & 30 & 0.3732 & $\checkmark$ \\
& 5 & 0.4571 & $\checkmark$ \\
\hline \multirow{2}{*}{2} & $1 \times 10^{-3}$ & 5.9937 & $\checkmark$ \\
& $5 \times 10^{-4}$ & 6.0381 & $\checkmark$ \\
\hline \multirow{2}{*}{3} & $3 \times 10^{-4}$ & 51.3714 & $\checkmark$ \\
& $1 \times 10^{-4}$ & 50.5591 & $\checkmark$ \\
\hline
\end{tabular}

We took the actual and estimated profiles of the bulk temperature at the feed side and the permeate sides as an example to illustrate the performance of the proposed observer. From Fig. 7 , it is clear that the proposed observer is able to follow the real states and the convergence is relatively fast. Furthermore, the Relative Error (RE) values for the state estimation are given in Table II. These values quantify the accuracy of the observer system compared to the actual system and it allows us to conclude on the performance of the proposed observer. Table II shows that for both temperature states and heat transfer rate states, the relative error is sufficiently small, which means that the observer estimates the system states with accuracy.

TABLE II: Relative error values for state estimation.

\begin{tabular}{cccccc}
\hline States & $T_{\mathrm{bf}_{1}}$ & $T_{\mathrm{bp}_{1}}$ & $T_{\mathrm{mf}_{1}}$ & $T_{\mathrm{mp}_{1}}$ & $T_{\mathrm{f}_{\text {out }}}$ \\
\hline RE & 0.0095 & 0.0197 & 0.0096 & 0.0143 & $8.8144 \times 10^{-6}$ \\
\hline States & $T_{\mathrm{p}_{\text {out }}}$ & $Q_{\mathrm{f}_{1}}$ & $Q_{\mathrm{f}_{2}}$ & $Q_{\mathrm{p}_{1}}$ & $Q_{\mathrm{p}_{2}}$ \\
\hline RE & $1.1053 \times 10^{-5}$ & 0.0028 & 0.0028 & 0.0989 & 0.0799 \\
\hline
\end{tabular}

\section{OBSERVER-BASED EMPC FOR DCMD}

In this section, we will integrate the nonlinear observer design of Eq. 7 for the DCMD process of Eq. 1 and the EMPC scheme developed in section III. In the first subsection, Observer-based EMPC (OEMPC) for DCMD process of Eq. 1 is introduced. In the second subsection, an implementation strategy that can ensure closed-loop stability of the proposed OEMPC is given.

\section{A. OEMPC Algorithm}

By combining the estimator and EMPC algorithm, we proposed the OEMPC algorithm, in which the following optimization problem is solved at every sampling time $t_{k}$ :

$$
\begin{aligned}
& \max _{u(t) \in S(\Delta)} \int_{t_{k}}^{t_{k+N}}\left[\beta_{1} J(\tilde{x}(\tau))-\beta_{2} u(\tau)\right] d \tau \\
& \text { s.t. } E \dot{\tilde{x}}=F(\tilde{x}, u) \tilde{x}+B(u) \\
& u(t) \in U, \forall t \in\left[t_{k}, t_{k+N}\right) \\
& \tilde{x}\left(t_{k}\right)=\hat{x}\left(t_{k}\right) \\
& G(\tilde{x}) \leq \rho_{e}, \forall t \in\left[t_{k}, t_{k+N}\right) . \\
&\left|u\left(t_{k}\right)-u\left(t_{k-1}\right)\right| \leq \varepsilon
\end{aligned}
$$

The above optimization problem is exactly the same as the EMPC formulation of Eq. 4 with only one modification. Specifically, for Eq. 17d, the initial state values for the prediction model of the EMPC will be equal to the estimated values derived from the nonlinear observer of Eq. 7 while the EMPC formulation of Eq. 4 assumes full knowledge of the state values for the DMCD process. The boundedness constraint of Eq. 17e ensures the closed-loop state within a pre-defined stability region. Hence the stability of the process is guaranteed by taking this boundedness constraint into account at the first sampling periods.

\section{B. Implement Algorithm}

The observer design of Eq. 7 receives the process measurements continuously and computes the estimates states based on the inputs $u^{*}(t)$ and $T_{\mathrm{f}_{\mathrm{in}}}(t)$. At each sampling time $t_{k}$, the OEMPC obtains the estimated states $\hat{x}\left(t_{k}\right)$ from the observer. Based on $\hat{x}\left(t_{k}\right)$, the OEMPC uses the process model of Eq. 1 to predict the future evolution of the system over the whole prediction horizon. Algorithm 1 summarizes the aforementioned implementation strategy. 


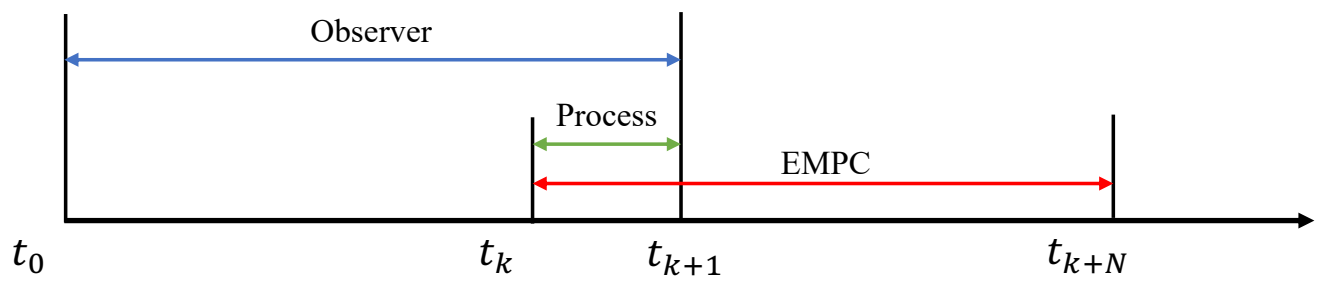

Fig. 8: Observer implementation strategy at sampling time $t_{k}$. Blue arrow refers to observer evaluating horizon (from $t_{0}$ to $t_{k+1}$ ); Green arrow is the evaluating horizon of the process model (from $t_{k}$ to $t_{k+1}$ ); Red arrow refers to the evaluating horizon of the EMPC (from $t_{k}$ to $t_{k+N}$ ).

It is worth to mention that since the DCMD process is modeled by a set of DAEs, the available DAE solver in MATLAB (e.g. ode15s) requires consistent initial conditions in order to successfully start the integration. Here the term consistent means that the initial condition cannot choose arbitrary like the Ordinary Differential Equations (ODEs) case, which should satisfy the algebraic equations defined in the system equations of Eq. 1.

However, since the observer system of Eq. 7 is also defined as a DAE system, which is different from the original DAE model of Eq. 1 due to the correction term in the state equation, we cannot simply adapt the solution of the predicted model within the EMPC as true consistent initial condition to the observer system. One way to overcome the issue is to choose the initialization of the DAE model for the observer from $t_{0}$ at every sampling time to make sure we start from a valid initial condition. Therefore, we just need to compute the consistent initial condition once for the observer system. Fig. 8 represents the above implementation strategy graphically.

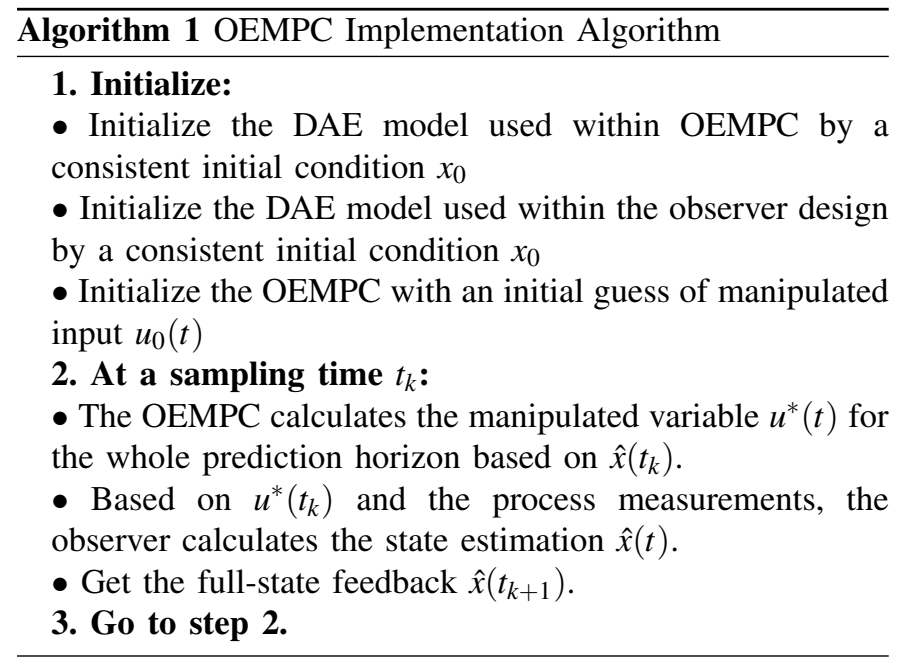

\section{Simulation RESUlts OF OEMPC}

In this section, we implement the OEMPC of Eq. 17 to the DCMD process of Eq. 1 for 6 hours (i.e. $t_{f}=6 \mathrm{~h}$ ). Throughout the simulation for OEMPC scheme, the number of controlvolume cells $\mathrm{N}_{c}$ chosen to model the system is 1 . This number of cells generates 10 differential and algebraic process states (6 differential states and 4 algebraic states). The feed inlet temperature is treated as a disturbance, the profile of $T_{\mathrm{f}_{\mathrm{in}}}$ is shown in Figure 3. The permeate inlet temperature $T_{\mathrm{p}_{\text {in }}}$ and the permeate inlet flow rate are fixed as $20^{\circ} \mathrm{C}$ and 0.1111 $\mathrm{m} / \mathrm{s}$, respectively. Due to the physical constraints on the solar thermal energy, the feed inlet mass flow rate $u(t)$ is constrained as follows $0 \mathrm{~m} / \mathrm{s} \leq u(t) \leq 2 \mathrm{~m} / \mathrm{s}$. The remaining model parameters used in this simulation are exactly the same as the ones used in [23].

The built in function in Matlab called ode15s with an integration step size of $h=10^{-3} \mathrm{~h}$ was used to simulate the DAE systems. As we discussed above, the DAE solver requires consistent initial conditions in order to successfully start the integration. Some methods for the initialization of DAE systems have been reported in [27]. In this paper, the consistent initial conditions are computed by finding the equilibrium point of the system. Specifically, we are solving the system's equations of Eq. 1 by setting the left hand side as zero (i.e. $0=F(x, u) x+B(u)$ ). The optimization problem of Eq. 17 was solved by the Matlab's built-in function fmincon. For the parameters of OEMPC used in the simulation, the prediction horizon $N$ is chosen as 10, the sampling period $\Delta$ is equal to $0.2 \mathrm{~h}$. The weighting parameters $\beta_{1}$ and $\beta_{2}$ are chosen as 1 and 2.5, respectively. Finally, the upper bound of the boundedness function $\rho_{e}$ is equal to $1.155 \times 10^{8}$.

In addition, since the observer is solved starting from $t_{0}$ at every sampling time, it is worth to check the efficiency of the observer solver. To do that, we study the computation time of the observer with different time period. Specifically, we investigate the computation time of observer system with time period $0.2 \mathrm{~h}, 3 \mathrm{~h}$, and $6 \mathrm{~h}$, respectively. After sufficient number of experiments, the following results are obtained: for $0.2 \mathrm{~h}$ time period, the mean of computation time is equal to $2.8 \mathrm{~s}$; the mean of the computation time under $3 \mathrm{~h}$ time period is equal to $3.0 \mathrm{~s}$; for $6 \mathrm{~h}$ time period, the mean of the computation time is equal to $2.65 \mathrm{~s}$. Therefore, we can conclude that for different range of time period, the observer solver can obtain the estimated states with almost equal computation time. For the real-time implementation, one can consider to define a sliding window for the proposed OEMPC algorithm. For example, we can define the length of sliding window equals to $6 \mathrm{~h}$, after 6 hours, the consistent initial condition can be computed again and the same strategy can be implemented to avoid the consistent initial condition issue as we discussed above.

Fig. 9 depicts the simulation results of the DCMD process of Eq. 1 under the OEMPC design of Eq. 17. Specifically, Fig. 9(a) to Fig. 9(c) show the closed-loop state trajectory and the 
manipulated input profile under the OEMPC scheme, respectively. One can observe that the manipulated input trajectory satisfied both the boundedness constraint of Eq. 17c and the smoothness constraint of Eq. 17f from Fig. 9(c). Based on this input profile, the system states are shown in Fig. 9(a) and Fig. 9(b). We separate the system states into two subplots due to the different magnitude of the system states. As shown in Fig. 9(a), the profile of temperature states at the feed side $T_{\mathrm{f}_{1}}$, $T_{\mathrm{mf}_{1}}$, and $T_{\mathrm{f}_{\text {out }}}$ follows the curve of the feed inlet temperature as shown in Fig. 3. However, the temperature states at the permeate sides $T_{\mathrm{p}_{1}}$ and $T_{\mathrm{p}_{\text {out }}}$ are close to $20^{\circ} \mathrm{C}$, which is the value we set for the permeate inlet temperature. Notice that $T_{m p_{1}}$ has the same trend as the feed inlet temperature but with smaller magnitude. This is mainly due to the fact that the heat transfer between the feed side and permeate side will affect more on the $T_{\mathrm{mp}_{1}}$ than $T_{\mathrm{bp}_{1}}$ and $T_{\mathrm{p}_{\text {out }}}$. The same phenomenon can be observed from Fig. 9(b). In this case, the profile of the heat transfer rate $Q_{\mathrm{f}_{1}}$ and $Q_{\mathrm{f}_{2}}$ at feed side is similar to the feed inlet mass flow rate as shown in Fig. 9(c). The curve of heat transfer rate $Q_{\mathrm{p}_{1}}$ and $Q_{\mathrm{p}_{2}}$ at permeate side are flat since we set the permeate inlet mass flow rate as constant. Fig. 9(d) shows the distilled water flux of the DCMD process of Eq. 1 under the OEMPC design of Eq. 17. It can be seen that as the inlet temperature increases, the distilled water flux $J$ rises. After achieving the maximum distilled water flux, it decreases as the feed inlet temperature decreases. We can also draw same conclusion from Eq. 3. Since the distilled water flux $J$ is calculated from $T_{\mathrm{mf}}$ and $T_{\mathrm{mp}}$, it is not surprising that the distilled water flux follows the structure of feed inlet temperature $T_{\mathrm{f}_{\mathrm{in}}}$ as shown in Fig. 3 since both $T_{\mathrm{mf}}$ and $T_{\mathrm{mp}}$ have similar trends.

In Fig. 10, the estimation error at every sampling time $t_{k}$ for each state is reported. It can be seen that at beginning, the estimator provides some inaccurate states. However, as time progresses, the estimation error starts to go to zero. This indicate that our observer implementation strategy can provides the accurate estimate states to initialize the prediction model within OEMPC. Since for each sampling period $\left(t_{k}, t_{k+1}\right]$, we solve the observer system start from $t_{0}$ (i.e. $\left[t_{0}, t_{k+1}\right]$ ). At first sampling period, the observer may not converge to the actual states since the convergence time for observer may larger than the sampling period of OEMPC. However, as more iteration evolves, the observer is able to converge to the actual system states with high accuracy.

Remark 1. The above simulation does not take measurement noise and process noise into account. When we simulate the closed-loop system with measurement noise, the inaccurate state estimation will cause some numerical issues in the simulations as the initial condition may not be consistent anymore. The consistent initial condition is necessary to integrate the DAE system equations successfully. If we consider both measurement noise and process noise, the situation becomes worse. Therefore to make our observer-EMPC scheme more robust, we can change our observer design to a robust version such as sliding mode or $H_{\infty}$ observer, for example. This may avoid the numerical issues due to non-consistent initial conditions and will help us to provide some guidelines on the
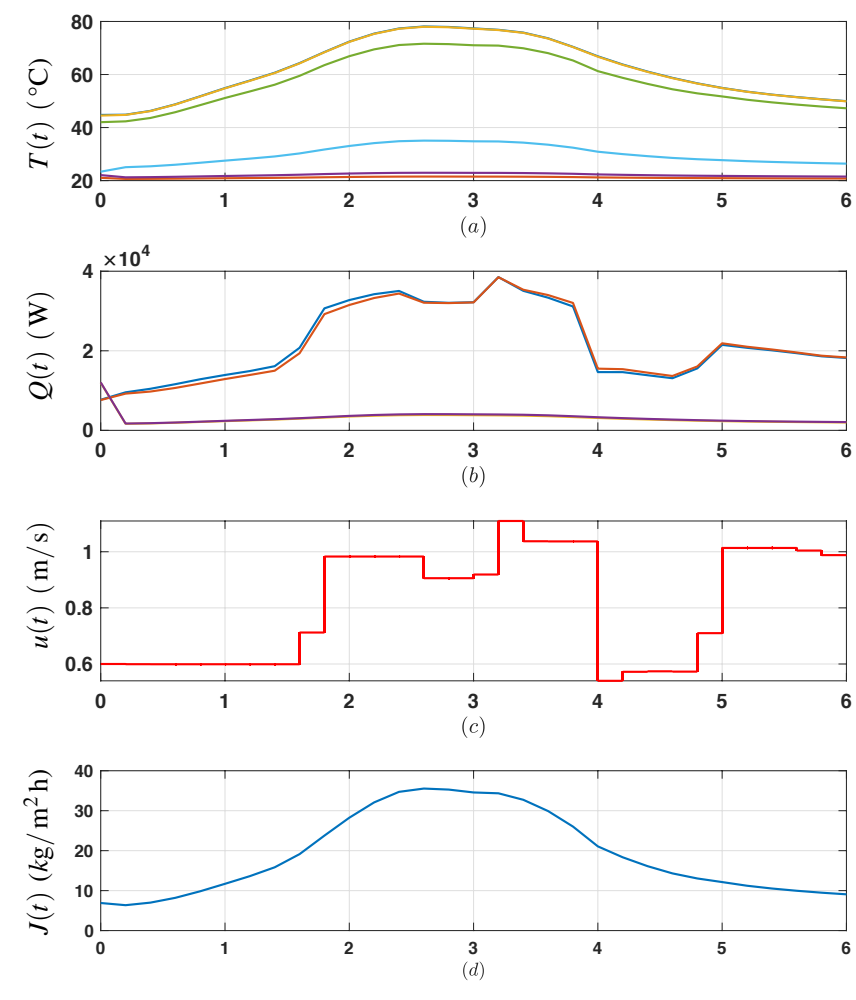

Time (h)

Fig. 9: Simulation results of DCMD under the OEMPC design (a): the temperature state trajectories $T(t)$, (b): the heat transfer rate state trajectories $Q(t)$, (c): the manipulated input profile $u(t)$, (d) the distilled water flux $J(t)$.

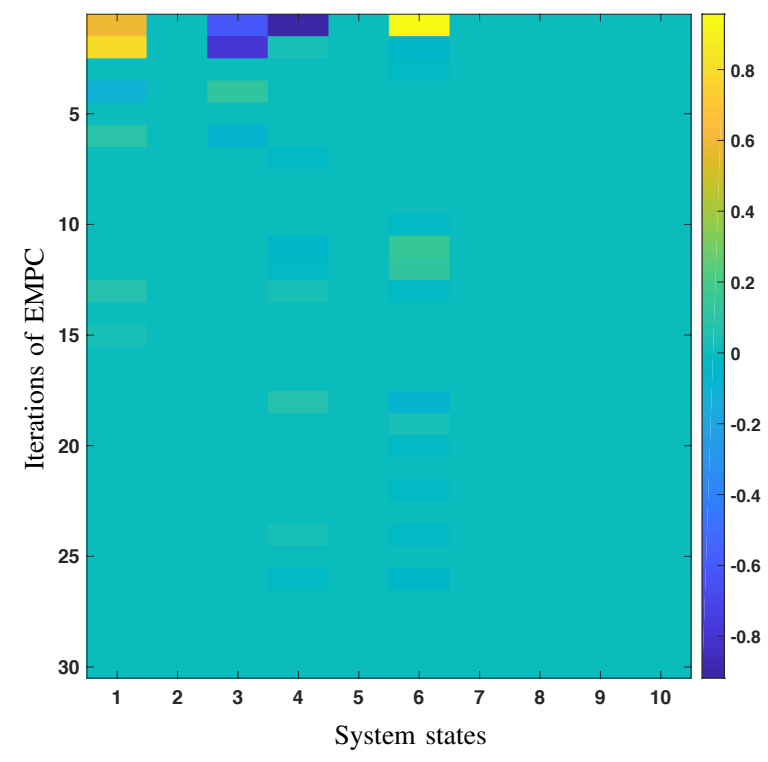

Fig. 10: Estimation error at each sampling time $t_{k}(k=1,2, \cdots)$ for each state along with the iterations of OEMPC. 
tolerance of our scheme to process and measurements noise.

\section{CONCLUSION}

In this paper, a unified framework of a nonlinear observer design and a Economic Model Predictive Control (EMPC), Observer-based EMPC (OEMPC), was proposed. For this framework, an implementation strategy that can ensure recursive feasibility and closed-loop stability of the OEMPC was given. The OEMPC was developed to maximize the distilled water flux while penalizing the feed inlet mass flow rate to operate the process economically. The nonlinear DAE-based observer was designed to calculate the unmeasured system states to initialize the DAE prediction model within OEMPC. Simulation results show that the estimate states converge to the actual states accurately. Finally, the closed-loop simulation results demonstrate that the OEMPC can optimally operate the DCMD process while meeting input and process constraints of the DCMD process. As for the future work, we will investigate the rigorous proof of the closed-loop stability of OEMPC as well as the real-time implementation.

\section{ACKNOWLEDGMENT}

Research reported in this publication has been supported by the King Abdullah University of Science and Technology (KAUST).

\section{REFERENCES}

[1] J. Åslund and E. Frisk. An observer for non-linear differential-algebraic systems. Automatica, 42:959-965, 2006.

[2] C.O. Ayala, L. Roca, J.L. Guzman, J.E. Normey-Rico, M. Berenguel, and L. Yebra. Local model predictive controller in a solar desalination plant collector field. Renewable Energy, 36:3001-3012, 2011.

[3] V. M. Becerra, P.D. Roberts, and G.W. Griffiths. Applying the extended kalman filter to systems described by nonlinear differential-algebraic equations. Control Engineering Practice, 9:267-281, 2001.

[4] P. Bendevis, A. Karam, and T.M. Laleg-Kirati. Optimal model-free control of solar thermal membrane distillation system. Computers \& Chemical Engineering, 2020.

[5] M. Boutayeb and M. Darouach. Observers design for nonlinear descriptor systems. In IEEE Conference on Decision and Control, 1995.

[6] T.C. Chen, C.D. Ho, and H.M. Yeh. Theoretical modeling and experimental analysis of direct contact membrane distillation. Journal of Membrane Science, 330(1-2):279287, 2009.

[7] A. Cipollina, M.G. Di Sparti, A. Tamburini, and G. Micale. Development of a membrane distillation module for solar energy seawater desalination. Chemical engineering research and design, 90:2101-2121, 2012.

[8] M. Darouach, M. Zasadzinski, and M. Hayar. Reducedorder observer design for descriptor systems with unknown inputs. IEEE transactions on automatic control, 41:1068-1072, 1996.
[9] A. Ebrahimi, G.D. Najafpour, and D.Y. Kebria. Performance of microbial desalination cell for salt removal and energy generation using different catholyte solutions. Desalination, 432:1-9, 2018.

[10] F. Eleiwi, N. Ghaffour, A.S. Alsaadi, L. Francis, and T.M. Laleg-Kirati. Dynamic modeling and experimental validation for direct contact membrane distillation (dcmd) process. Desalination, 384:1-11, 2016.

[11] F. Eleiwi and T.M. Laleg-Kirati. Nonlinear observerbased lyapunov boundary control of distributed heat transfer mechanisms for membrane distillation plant. Journal of Process Control, 47:78-86, 2016.

[12] F. Eleiwi and T.M. Laleg-Kirati. Observer-based perturbation extremum seeking control with input constraints for direct-contact membrane distillation process. International Journal of Control, 91:1363-1375, 2018.

[13] M. Ellis, H. Durand, and P.D. Christofides. A tutorial review of economic model predictive control methods. Journal of Process Control, 24:1156-1178, 2014.

[14] M. Ellis, J. Liu, and P.D Christofides. Economic Model Predictive Control, chapter State Estimation and EMPC, pages 135-170. Springer, 2017.

[15] C. Gera. Differential-algebraic equations, indices, and integral algebraic equations. SIAM Journal on Numerical Analysis, 27:1527-1534, 1990.

[16] M. Ghattassi, J.C. Vivalda, and T. M. Laleg-Kirati. State observer design for direct contact membrane distillation parabolic systems. In Annual American Control Conference, Milwaukee, June. 2018.

[17] D.J. Gila, L. Roca, A. Ruiz-Aguirre, G. Zaragoza, and M. Berenguel. Optimal operation of a solar membrane distillation pilot plant via nonlinear model predictive control. Computers \& Chemical Engineering, 109:151165, 2018.

[18] X.G. Guo, F. Albalawi, and T.M. Laleg-Kirati. Model predictive control paradigms for direct contact membrane desalination modeled by differential algebraic equations. In American Control Conference (ACC), pages 55955601, 2019.

[19] E. Hairer, C. Lubich, and M. Roche. The numerical solution of differential-algebraic systems by runge-kutta methods. Springer Berlin Heidelberg, 1989.

[20] M. Heidarinejad, J. Liu, and P.D. Christofides. Stateestimation-based economic model predictive control of nonlinear systems. Systems \& Control Letters, 61:926935, 2012.

[21] M. Hou and P.C. Muller. Observer design for descriptor systems. IEEE Transactions on Automatic Control, 44:164-169, 1999.

[22] A.M. Karam, A.S. Alsaadi, N. Ghaffour, and T.M. LalegKirati. Analysis of direct contact membrane distillation based on a lumped-parameter dynamic predictive model. Desalination, 402:50-61, 2017.

[23] A.M. Karam and T.M. Laleg-Kirati. Electrical equivalent thermal network for direct contact membrane distillation modeling and analysis. Journal of Process Control, 47:87-97, 2016.

[24] L. Lao, M. Ellis, H. Durand, and P.D. Christofides. Real- 
time preventive sensor maintenance using robust moving horizon estimation and economic model predictive control. AIChE Journal, 61(10):3374-3389, 2015.

[25] J. Lofberg. Yalmip : a toolbox for modeling and optimization in matlab. IEEE Conference on Robotics and Automation, pages 284-289, 2004.

[26] G. Lu and D. Ho. Full-order and reduced-order observers for lipschitz descriptor systems: the unified lmi approach. IEEE Transactions on Circuits and Systems II: Express Briefs, 53:563-567, 2006.

[27] N.B. Peter, A.C. Hindmarsh, and L.R. Petzold. Consistent initial condition calculation for differential-algebraic systems. SIAM Journal on Scientific Computing, 19:1495-1512, 1998.

[28] L. Song, B. Li, K.K. Sirkar, and J.L. Gilron. Direct contact membrane distillation-based desalination: novel membranes, devices, larger-scale studies, and a model. Industrial and engineering chemistry research, 46(23072323), 2007.

[29] E. Uezato and M. Ikeda. Strict Imi conditions for stability, robust stabilization, and $\mathrm{H}$ infinity control of descriptor systems. In IEEE Conference on Decision and Control, pages 4092-4097, 1999.

[30] G. Zaragoza, A. Ruiz-Aguirre, and E. Guillén-Burrieza. Efficiency in the use of solar thermal energy of small membrane desalination systems for decentralized water production. Applied Energy, 130:491-499, 2014.

\section{APPENDIX A}

\section{NOMENCLATURE}

List of notations:

\begin{tabular}{|c|c|c|}
\hline Symbol & Description & Unit \\
\hline$A_{m}$ & Differential cell membrane area & $\left(\mathrm{m}^{2}\right)$ \\
\hline$a$ & Model parameters & \\
\hline$\Delta$ & Sampling time of OEMPC & ( h) \\
\hline$h$ & Integration step & (h) \\
\hline$J$ & Mass flux of distilled water & $\left(\mathrm{kg} / \mathrm{m}^{2} \mathrm{~s}\right)$ \\
\hline$M$ & Mass flow rate & $(\mathrm{m} / \mathrm{s})$ \\
\hline$N$ & Predict horizon of OEMPC & \\
\hline$Q$ & Heat transfer rate & Watt (W) \\
\hline$R$ & Thermal resistance & $\left({ }^{\circ} \mathrm{C} / \mathrm{W}\right)$ \\
\hline$T$ & Temperature & $\left({ }^{\circ} \mathrm{C}\right)$ \\
\hline$t_{0}$ & Initial simulation time of OEMPC & (h) \\
\hline$t_{f}$ & Final simulation time of OEMPC & (h) \\
\hline Subscripts & Description & Unit \\
\hline $\mathrm{b}$ & Bulk & \\
\hline $\mathrm{f}$ & Feed & \\
\hline in & Inlet & \\
\hline $\mathrm{m}$ & Membrane & \\
\hline $\mathrm{n}$ & Index for cell number & \\
\hline$N_{c}$ & Total number of cells & \\
\hline out & Outlet & \\
\hline $\mathrm{p}$ & Permeate & \\
\hline Greek letters & Description & Unit \\
\hline$\alpha$ & Model parameter & \\
\hline$\beta$ & Tuning parameter & \\
\hline$\delta$ & Membrane mass transfer coefficient & \\
\hline$\varepsilon$ & Smoothness constraint upperbound & $(\mathrm{m} / \mathrm{s})$ \\
\hline$\gamma$ & Lipschitz constant & \\
\hline$\dot{\lambda}$ & Mole fraction of $\mathrm{NaCl}$ in the feed stream & \\
\hline$\rho_{e}$ & Upper bound of the boundedness function & \\
\hline
\end{tabular}

\section{APPENDIX B \\ DAE MODEL OF DCMD}

In this Appendix section, the expressions of matrix $\mathrm{A}$ and $\Phi(x, u)$ is given. As mentioned in section IV, the DAE model of DCMD of Eq. 1 can be represented as the following:

$$
E \dot{x}(t)=A x(t)+B(u)+\Phi(x, u)
$$

where $x(t) \in \Omega \subset R^{6 \mathrm{~N}_{c}+4}$ is the system states, $A, E \in$ $R^{6 \mathrm{~N}_{c}+4 \times 6 \mathrm{~N}_{c}+4}$ and $C \in R^{2 \times 6 \mathrm{~N}_{c}+4}$ are constant matrices; $\Phi(t, x) \in R^{6 \mathrm{~N}_{c}+4}$ is the nonlinear part of the DCMD model. Due to the space limitation, here we present the system equations when $\mathrm{N}_{c}=1$ as shown from Eq. 18 to Eq. 27. By separating the linear part and nonlinear part from these equations, we get the A matrix and nonlinear equations $\Phi(x, u)$ as follows:

$A=\left[\begin{array}{ccccc}\mathscr{L}\left(A_{f}(x, u)\right) & \mathbf{0} & \mathbf{0} & Z_{f_{2}} & \mathbf{0} \\ \mathbf{0} & \mathscr{L}\left(A_{p}(x, u)\right) & \mathbf{0} & \mathbf{0} & \mathscr{L}\left(Z_{p_{2}}(x)\right) \\ \mathbf{0} & \mathbf{0} & I & \mathbf{0} & \mathbf{0} \\ \mathscr{L}\left(Z_{1}(x)\right) & Z_{2} & \mathbf{0} & Z_{3} & \mathscr{L}\left(Z_{4}(x)\right) \\ \mathbf{0} & Z_{5} & \mathbf{0} & \mathscr{L}\left(Z_{6}(x)\right) & \mathscr{L}\left(Z_{7}(x)\right)\end{array}\right]$,

where the operator $\mathscr{L}($.$) means to take the linear part of the$ evaluating block matrix and:

$A_{\mathrm{f}}(x, u) \in R^{2 N_{c}+1 \times 2 N_{c}+1}:$ Tridiagonal matrix representing the feed differential dynamics.

$A_{\mathrm{p}}(x, u) \in R^{2 N_{c}+1 \times 2 N_{c}+1}$ : Tridiagonal matrix representing the permeate differential dynamics.

$Z_{\mathrm{f}_{2}} \in R^{2 N_{c}+1 \times N_{c}}$ : Coupling to the membrane-feed interface.

$Z_{\mathrm{p}_{2}}(x) \in R^{2 N_{c}+1 \times N_{c}}:$ Coupling to the membrane-permeate interface.

$I \in R^{2 \times 2}$ : The identity matrix.

$Z_{1}(x) \in R^{N_{c} \times 2 N_{c}+1}:$ Sparse matrix.

$Z_{2} \in R^{N_{c} \times 2 N_{c}+1}:$ Sparse matrix.

$Z_{3} \in R^{N_{c} \times N_{c}}$ : Diagonal matrix.

$Z_{4}(x) \in R^{N_{c} \times N_{c}}$ : Diagonal matrix.

$Z_{5} \in R^{N_{c} \times 2 N_{c}+1}:$ Sparse matrix.

$Z_{6}(x) \in R^{N_{c} \times N_{c}}$ : Diagonal matrix.

$Z_{7}(x) \in R^{N_{c} \times N_{c}}$ : Diagonal matrix.

Specifically, we have:

$$
\begin{gathered}
\mathscr{L}\left(A_{f}(x, u)\right)=\left[\begin{array}{ccc}
-4 a_{1} M_{\mathrm{f}_{\mathrm{in}}}^{2} & -8 a_{2} M_{\mathrm{f}_{\mathrm{in}}}^{4} & 0 \\
a_{5} & -a_{4} & -a_{5} \\
0 & 0 & 0
\end{array}\right], \\
\mathscr{L}\left(A_{p}(x, u)\right)=\left[\begin{array}{ccc}
0 & 0 & 0 \\
a_{9} & -a_{8} & -a_{9} \\
0 & 0 & 0
\end{array}\right] \\
Z_{\mathrm{f}_{2}}=\left[\begin{array}{lll}
0 & a_{4} & 0
\end{array}\right]^{\mathrm{T}} \\
\mathscr{L}\left(Z_{\mathrm{p}_{2}}(x)\right)=\left[\begin{array}{lll}
0 & a_{8} & 0
\end{array}\right]^{\mathrm{T}} \\
\mathscr{L}\left(Z_{1}(x)\right)=\left[\begin{array}{lll}
0 & \frac{1}{R_{\mathrm{f}}} & 0
\end{array}\right]
\end{gathered}
$$


System equations with $\mathrm{N}_{c}=1$ :

$$
\begin{aligned}
\dot{Q}_{f_{1}} & =-4 a_{1} M_{f_{\text {in }}}^{2} Q_{f_{1}}-8 a_{2} M_{f_{\text {in }}}^{4} T_{b f_{1}} \\
\dot{T}_{b_{f_{1}}} & =a_{5} Q_{f_{1}}+\left(-a_{3} J_{1}-a_{4}\right) T_{b_{f_{1}}}-a_{5} Q_{f_{2}}+a_{4} T_{m f_{1}} \\
\dot{Q}_{f_{2}} & =4 a_{2} M_{f_{1}}^{2} T_{b f_{1}}-4 a_{1} M_{f_{1}}^{2} Q_{f_{2}}-8 a_{2} M_{f_{1}}^{4} T_{f_{\text {out }}} \\
\dot{Q}_{p_{1}} & =-4 a_{6} M_{p_{1}}^{2} Q_{p_{1}}-8 a_{7} M_{p_{1}}^{2} T_{b p_{1}}+8 a_{7} M_{P_{1}}^{4} T_{p_{\text {out }}} \\
\dot{T}_{b_{p_{1}}} & =a_{9} Q_{p_{1}}-a_{8} T_{b p_{1}}-a_{9} Q_{p_{2}}+\left(a_{10} J_{1}+a_{8}\right) T_{m p_{1}} \\
\dot{Q}_{p_{2}} & =4 a_{7} M_{p_{2}}^{4} T_{b p_{1}}-4 a_{6} M_{p_{\text {in }}}^{2} Q_{p_{2}} \\
0 & =-\frac{1}{M_{f_{1}} c_{p}} Q_{f_{2}}+T_{f_{\text {out }}} \\
0 & =\frac{1}{M_{p_{1}} c_{p}} Q_{p_{1}}+T_{p_{\text {out }}} \\
0 & =\left(\frac{1}{R_{f}}+a_{11} J_{1}\right) T_{b f_{1}}+\frac{1}{R_{p}} T_{b p_{1}}-\frac{1}{R_{f}} T_{m f_{1}}+\left(-\frac{1}{R_{p}}-a_{11} J_{1}\right) T_{m p_{1}} \\
0 & =-\frac{1}{R_{p}} T_{b p_{1}}+\left(-\frac{1}{R_{m}}-A_{m} H_{v}\left[T_{m f_{1}}\right] J_{1}\right) T_{m f_{1}}+\left(\frac{1}{R_{m}}+\frac{1}{R_{p}}+a_{11} J_{1}\right) T_{m p_{1}}
\end{aligned}
$$

$$
\begin{aligned}
& Z_{2}=\left[\begin{array}{lll}
0 & \frac{1}{R_{\mathrm{p}}} & 0
\end{array}\right], \\
& Z_{5}=\left[\begin{array}{lll}
0 & -\frac{1}{R_{\mathrm{p}}} & 0
\end{array}\right],
\end{aligned}
$$

for different scenarios. The submatrix $L_{a 1}$ of gain matrix $L$ have been bolded, one can easily check the rank condition of Eq. 16 is satisfied. In the simulation of section VI, we choose the gain matrix $L$ under the condition $\gamma=30$ and $\mathrm{N}_{c}=1$.

$$
\left[\begin{array}{cc}
Z_{3} & \mathscr{L}\left(Z_{4}(x)\right) \\
\mathscr{L}\left(Z_{6}(x)\right) & \mathscr{L}\left(Z_{7}(x)\right)
\end{array}\right]=\left[\begin{array}{cc}
-\frac{1}{R_{\mathrm{f}}} & -\frac{1}{R_{\mathrm{p}}} \\
-\frac{1}{R_{\mathrm{m}}} & \frac{1}{R_{\mathrm{m}}}+\frac{1}{R_{\mathrm{p}}}
\end{array}\right] .
$$

where $M_{\mathrm{f}_{\mathrm{in}}}$ refers to the feed inlet mass flow rate, which is a constant in our case; the rest parameters appears above are also can be treated as constants which were reported in [23].

For the nonlinear part, we have:

$$
\Phi(x, t)=\left[\begin{array}{c}
0 \\
-a_{3} J T_{\mathrm{b}_{\mathrm{f}_{1}}} \\
4 a_{2} M_{\mathrm{f}_{1}}^{2} T_{\mathrm{bf}_{1}}-4 a_{1} M_{\mathrm{f}_{1}}^{2} Q_{\mathrm{f}_{2}}-8 a_{2} M_{\mathrm{f}_{1}}^{4} T_{\mathrm{f}_{\text {out }}} \\
-4 a_{6} M_{\mathrm{p}_{1}}^{2} Q_{\mathrm{p}_{1}}-8 a_{7} M_{\mathrm{p}_{1}}^{2} T_{\mathrm{bp}_{1}}+8 a_{7} M_{\mathrm{p}_{1}}^{4} T_{\mathrm{p}_{\text {out }}} \\
a_{10} J T_{\mathrm{mp}_{1}} \\
4 a_{7} M_{\mathrm{p}_{2}}^{4} T_{\mathrm{bp}_{1}} \\
-\frac{1}{M_{\mathrm{f}_{1}} c_{p}} Q_{\mathrm{f}_{2}} \\
\frac{1}{M_{\mathrm{p}_{1}} c_{p}} Q_{\mathrm{p}_{1}} \\
a_{11} J T_{\mathrm{bf}_{1}}-a_{11} J T_{\mathrm{mp}_{1}} \\
-A_{m} H_{v}\left[T_{\mathrm{mf}_{1}}\right] J T_{\mathrm{mf}_{1}}+a_{11} J T_{\mathrm{mp}_{1}}
\end{array}\right]
$$

where

$$
\begin{gathered}
M_{\mathrm{f}_{1}}=M_{\mathrm{f}_{\mathrm{in}}}-A_{m} J, \\
M_{\mathrm{p}_{1}}=M_{\mathrm{P}_{\text {in }}}+A_{m} J, \\
H_{v}[T]=-2.426 T+2503 .
\end{gathered}
$$

\section{APPENDIX C}

\section{OBSERVER GAIN MATRIX}

In this section, we report the observer gain matrix used in this work. In section IV, we compute the observer gain matrix offline by computing several LMIs with different Lipschitz constants $\gamma$. Specifically, we got the following gain matrices 
Observer gain matrices with different $\gamma$ and $\mathrm{N}_{c}$ :

$$
\begin{aligned}
& \gamma=30, \mathrm{~N}_{c}=1, L=\left[\begin{array}{cccccccccc}
0.18 & 0.20 & 0.16 & 0.28 & 2.46 & 0.10 & \mathbf{2 . 3} \times \mathbf{1 0}^{\mathbf{1 4}} & \mathbf{2 . 3} \times \mathbf{1 0}^{\mathbf{1 2}} & 9.60 & -10.54 \\
-0.40 & -0.47 & -0.46 & -0.37 & 2.54 & -0.58 & \mathbf{7 . 6} \times \mathbf{1 0}^{\mathbf{1 2}} & \mathbf{2 . 4} \times \mathbf{1 0}^{\mathbf{1 4}} & 9.46 & -10.37
\end{array}\right]^{\mathrm{T}}, \\
& \gamma=5, \mathrm{~N}_{c}=1, L=\left[\begin{array}{llllllllll}
-20.52 & -3.17 & -20.39 & -22.39 & -86.30 & -18.59 & \mathbf{1 . 1} \times \mathbf{1 0}^{\mathbf{1 3}} & \mathbf{3 . 0} \times \mathbf{1 0}^{\mathbf{1 3}} & -1225.63 & 307.58 \\
-20.84 & -3.46 & -20.68 & -22.62 & -86.24 & -18.87 & \mathbf{2 . 9} \times \mathbf{1 0}^{\mathbf{1 3}} & \mathbf{1 . 3} \times \mathbf{1 0}^{\mathbf{1 3}} & -1224.38 & 307.22
\end{array}\right]^{\mathrm{T}}, \\
& \gamma=1 \times 10^{-3}, \mathrm{~N}_{c}=2, L=\left[\begin{array}{ccccccccc}
-28.95 & 121.34 & -17.13 & -1579.51 & -20.87 & -16723.84 & 9791.44 & -90233.59 & \ldots \\
-28.45 & -39.23 & -18.11 & -1418.94 & -20.38 & -25240.17 & 1192 & -73200.88 & \ldots
\end{array}\right. \\
& \left.\begin{array}{lccccccc}
-81300.17 & 106890.45 & \mathbf{4 . 7} \times \mathbf{1 0}^{\mathbf{1 1}} & \mathbf{- 0 . 0 1} & 0.07 & -0.63 & -5565.52 & 47891.64 \\
-72700.72 & 98374.13 & -\mathbf{0 . 0 1} & \mathbf{4 . 7} \times \mathbf{1 0}^{\mathbf{1 1}} & 0.01 & -0.57 & -518.93 & 4.3 \times 10^{4}
\end{array}\right]^{\mathrm{T}}, \\
& \gamma=5 \times 10^{-4}, \mathrm{~N}_{c}=2, L=\left[\begin{array}{ccccccccc}
-22.22 & 9105.16 & 51.58 & -5231.83 & 13.42 & 548497.41 & 479954.71 & -758090.27 & \ldots \\
21.09 & 9826.21 & 53.85 & -5952.88 & 12.29 & 586625.38 & 518409.44 & -834346.19 & \ldots
\end{array}\right. \\
& \left.\begin{array}{cccccccc}
-284661.77 & 209680.08 & \mathbf{8 . 0} \times \mathbf{1 0}^{\mathbf{1 1}} & \mathbf{0} & 6.49 & -3.83 & -282149.27 & 166559.09 \\
-323116.5 & 247808.04 & \mathbf{0} & \mathbf{8 . 0} \times \mathbf{1 0}^{\mathbf{1 1}} & 7.01 & -4.35 & -304716.08 & 189125.90
\end{array}\right]^{\mathrm{T}}, \\
& \gamma=3 \times 10^{-4}, \mathrm{~N}_{c}=3, L=\left[\begin{array}{lllllllll}
412.74 & -48156.44 & 169.63 & 58284.44 & 305.99 & 36029.55 & 337.42 & -1231351.17 & \ldots \\
415.18 & -50765.92 & 163.36 & 60631.05 & 307.41 & 36261.79 & 339.40 & -1319298.94 & \ldots
\end{array}\right. \\
& \begin{array}{ccccccccc}
-1756265.06 & 4207297.44 & 2036359.35 & -623970.41 & 1228288.13 & -2350750.04 & \mathbf{6 . 6} \times \mathbf{1 0}^{\mathbf{1 1}} & \mathbf{0} & \ldots \\
-1849326.68 & 4386949.12 & 2120060.20 & -682687.13 & 1235970.68 & -2383737.63 . & \mathbf{0} & \mathbf{6 . 6} \times \mathbf{1 0} & \ldots
\end{array} \\
& \left.\begin{array}{llllll}
-8.63 & 10.12 & 6.2 & 1021124.39 & -1197570.70 & -733097.02 \\
-9.09 & 10.54 & 6.24 & 1075422.11 & -1246431.09 & -738013.32
\end{array}\right]^{\mathrm{T}} \\
& \gamma=1 \times 10^{-4}, \mathrm{~N}_{c}=3, L=\left[\begin{array}{lllllllll}
13.31 & 5506.18 & 13.97 & 5397.20 & 16.62 & 1319.68 & 11.51 & 361976.17 & \ldots \\
13.18 & 5669.02 & 14.05 & 5464.14 & 16.80 & 1087.80 & 11.37 & 369557.20 & \ldots
\end{array}\right. \\
& \begin{array}{llllllllll}
189915.14 & 44093.75 & 185875.87 & -222327.02 & 41433.39 & -183687.52 & \mathbf{3 . 4} \times \mathbf{1 0}^{\mathbf{1 1}} & \mathbf{0} & \ldots
\end{array} \\
& \begin{array}{lllllllll}
195671.07 & 39112.42 & 188268.85 & -234153.05 & 33189.69 & -174461.20 & \mathbf{0} & \mathbf{3 . 4} \times \mathbf{1 0}^{\mathbf{1 1}} & \ldots
\end{array} \\
& \left.\begin{array}{llllll}
0.50 & 0.49 & 0.11 & -112895.20 & -110907.47 & -25623.09
\end{array}\right]^{\mathrm{T}} \\
& \left.\begin{array}{llllll}
0.51 & 0.49 & 0.09 & -116289.73 & -112304.10 & -20789.16
\end{array}\right]
\end{aligned}
$$

